\title{
Textiles as Material Gestalt: Cloth as a Catalyst in the Co-designing Process
}

\section{Katherine Townsend $(\mathbb{D}$ and Ania Sadkowska}

Katherine Townsend is an associate professor in Fashion and Textile Crafts in the School of Art and Design at Nottingham Trent University. She has a background in the fashion and textile industry where she co-directed two independent labels based and worked as a freelance designer. Her current research projects, "Emotional Fit: Developing a new fashion methodology with mature women" and "The Electric Corset and Other Future Histories", explore material and cultural issues in fashion, textiles and ageing and the use of costume archives to inform the design of e-textiles and wearables. Her research into the role of embodied knowledge in the digital crafting of textiles was recently published in the chapter Closely Held Secrets, in Crafting Textiles in the Digital Age (Bloomsbury 2016). She is co-editor of the journal of Craft Research, Intellect.

katherine.townsend@ntu.ac.uk

Ania Sadkowska is a fashion designer, lecturer and researcher at Coventry University, United Kingdom. In the past, she worked as a Lecturer and Research Fellow at Nottingham Trent University, United Kingdom. Her research explores the intersection of sociology and psychology with art and design
ABSTRACT Textiles is the common language within Emotional Fit, a collaborative research project investigating a person-centred, sustainable approach to fashion for an ageing female demographic (55+). Through the co-designing of a collection of research tools, textiles have acted as a material gestalt for exploring our research participants' identities by tracing their embodied knowledge of fashionable dress. The methodology merges Interpretative Phenomenological Analysis, co-design and a simultaneous approach to textile and garment design. Based on an enhanced understanding of our participants textile preferences, particular fabric qualities have catalysed silhouettes, through live draping and geometric pattern cutting to accommodate multiple body shapes and customisation. Printed textiles have also been digitally crafted in response to the contours of the garment and body and personal narratives of wear. Sensorial and tactile interactions have informed the engineering and scaling of patterns within zero-waste volumes. The article considers the functional and aesthetic role of textiles 
practices. Current projects span a variety of topics including fashion and ageing, masculinity, phenomenology, and art and design research methodologies. Ania has presented work at various UK and international conferences and exhibitions including Italy, China, Sweden and United States. Recently her research paper has been published in the Age, Culture, Humanities: An Interdisciplinary Journal (special issue on Fashion and Ageing). Since 2014, she has been involved in a co-creative research project titled "Emotional Fit: Developing a new fashion methodology with older women".

ac3739@coventry.ac.uk through the co-creative development of printed garment prototypes that explore the physical and emotional aspects of fashion and ageing. Within the practice-led research, emphasis is placed on the participants involvement through manipulating and animating emerging textile and dress objects. The collaborative exchange draws on fundamental connections between dress and personal identity, utilizing cloth to mediate material and individual agency. The methodology seeks to capture and enact values attributed to material engagement through body-cloth dialogue. By reflecting on the longevity of particular items of dress we investigate how textile attachment can inform emotionally durable fashion solutions.

KEYWORDS: participatory research, embodied knowledge, co-creation, emotional fit, textile attachment, material engagement

\section{Introduction}

Emotional Fit is an ongoing, collaborative research project which is developing a new, more holistic approach to fashion design for older consumers by engaging with them in the co-creative and socio-material process of making (Townsend et al. 2017b). By working with a group of over 40 women from Nottingham, aged 55+, we are exploring some of the physical and psychological issues that concern these individuals, who are all part of the baby boomer generation (Gilleard and Higgs 2014). In this article we discuss some of the collaborative designing and making aspects involved in the project and how our participants' embodied understanding of fashion is strongly connected to the relationship between body image and the semantics of cloth; we focus particularly on how textiles act as a catalyst in their past and future lives in relation to their personalised self-identity and image (Kent 2017).

The project was developed following a request from the participating group, who communicated their dissatisfaction with the current high street fashion offer to researchers in the School of Art and Design, Nottingham Trent University, leading to a co-creative investigation. The project was initiated in 2015 by Townsend with Sadkowska, practice-led researchers in fashion and textiles (e.g. Townsend 2004, 2011, 2016) and fashion and ageing (Sadkowska 2016; Sadkowska et al. 2016) in collaboration with pattern cutter and knitwear designer, Juliana Sissons (2010). In 2016 senior fashion design lecturer, Karen Harrigan and research fellow, Katherine West joined the team. While each researcher was nominally assigned a particular role at the outset, based on their specialist knowledge and skills, as the project developed and grew, sensibilities and practices overlapped. The roles of the researchers and participants also blurred, resulting in everyone being engaged in the co-designing of the final experimental womenswear prototypes. The methodology, whereby fashion and textile design are practiced with as opposed to for potential consumers, offers a fresh take on DIY and slow fashion and their value to society (von Bush 2009; Townsend et al. 2016; Twigger Holroyd 2017a).

The core aim of the research is to investigate the source of older women's dissatisfaction with contemporary clothing by developing 
understanding of the perceived issues through a co-designing project. The temporary and mutable nature of "fashion" make it difficult to define and research, but "in spite of being ephemeral it is indexed in material form" (Woodward and Fisher 2014: 3). In this vein, by investigating items from the participant's wardrobes as a starting point for a series of workshops and interviews, the researchers sought to understand their relationship with contemporary "dress objects" (Skjold 2016: 136), and how the "clothing and person were entangled" (Woodward and Fisher 2014: 5). The term "dress" is used here to define fashion as a form of universal bodily adornment (Benthall 1976; Polhemus and Proctor 1978, cited in Entwistle 2015: 42) with focus on the meanings and practices associated with particular garments (Barnes and Eicher 1992, cited in Entwistle 2015: 42). "Dress" also suggests the act of dressing and covering the body encompassing the aesthetic concept of adornment [as] the choices made in getting dressed can be aesthetic as much as 'functional'" (Entwistle 2015: 43), aspects of which we discuss here.

The article is organized into two main sections, reflecting on Understanding (Phase I) our participants experiences of textiles and dress, then acting on findings through Co-designing and Production (Phases II and III) as illustrated in Table 1. Section 2 summarises the Emotional Fit Methodology and its triangulation of interpretative phenomenal analysis (IPA), participatory/ co-design and simultaneous design (SDM) methods (Townsend et al. 2017b). Tracing Participants Embodied Knowledge of Textiles and Dress (2.1) presents examples of how textile attachment informs "emotional durability" (Chapman 2014) derived from interview analysis. The 'textiles as a catalyst' master theme identified through the IPA process (Table 2) is interpreted through two sub-themes: Textiles as

Table 1 The Emotional Fit Research Model June 2015-April 2017 @Emotional Fit 2015

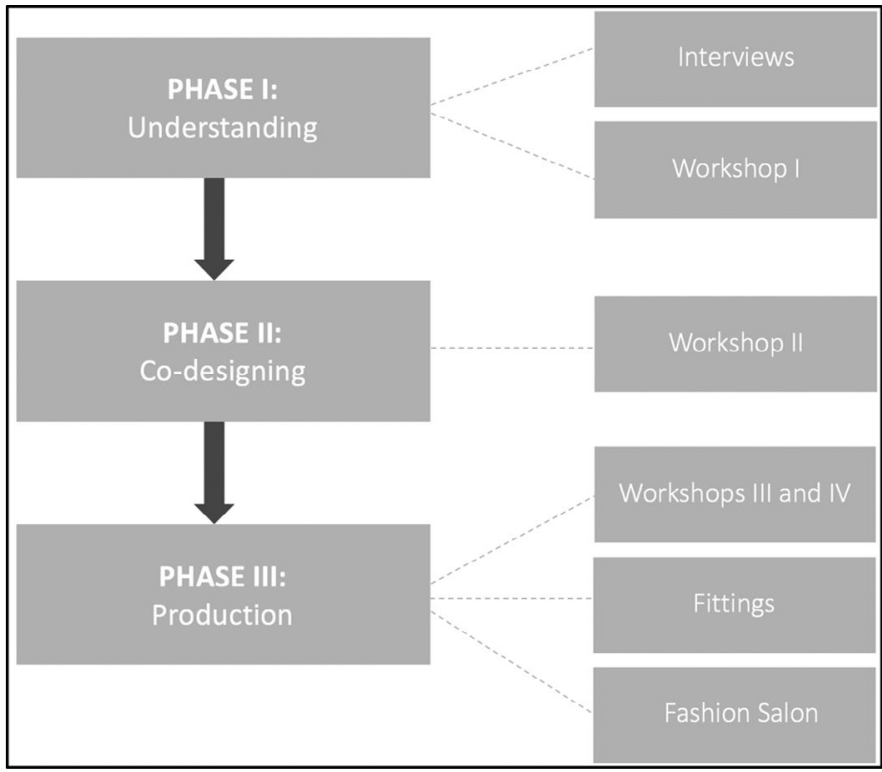


Material Gestalt (2.2) which connects "material engagement" (Malafouris 2013) with individual agency; and by considering Surface Pattern as Embodied Narrative and Emotional Expression (2.3). Section 3 explores The Interrelating of Print, Fabric and Form based on interview findings and co-creative practice and How Textile Preferences Informed Garment Silhouettes (3.1) in terms of aesthetic and zero waste (Rissanen and McQuillan 2015) criteria. Bespoke prints were created by Connecting Past and Future Textile Narratives (3.2) followed by a process of Engineering and Constructing Printed Textiles as Research Tools (3.3) resulting in "in-between" (Aakko 2016) garments, adapted in response to the feedback following trying on exercises, in private and public settings. Section 4 provides a Discussion of the key points arising from focusing on the significant role of textiles within emotionally durable fashion design. Figure 1 features an image from the Emotional Fit (2017) fashion salon, where the co-created designs were modelled by the participants.

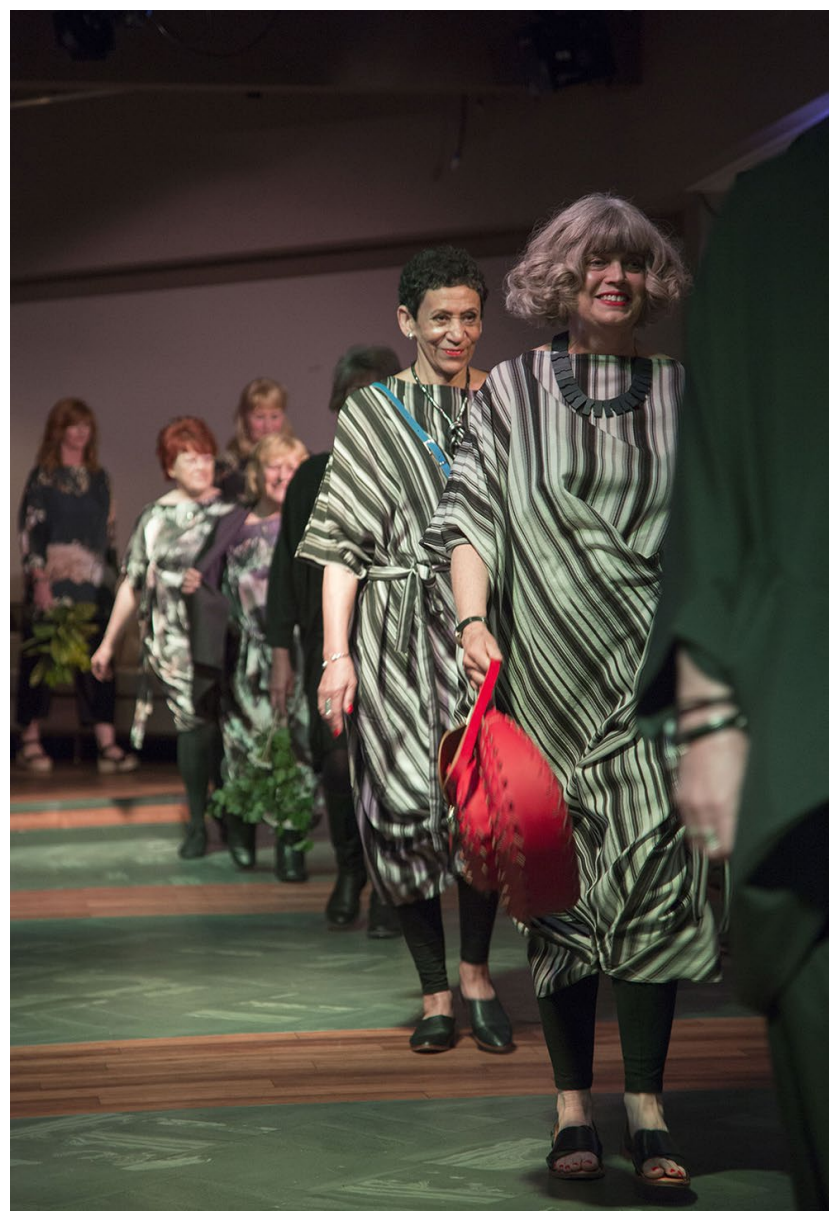

Figure 1

Photograph of the Emotional Fit Fashion Salon, a public event held at Antenna, Nottingham in April 2017, where participants modeled garment prototypes they had co-designed (c) Rebecca Lewis 2017. 

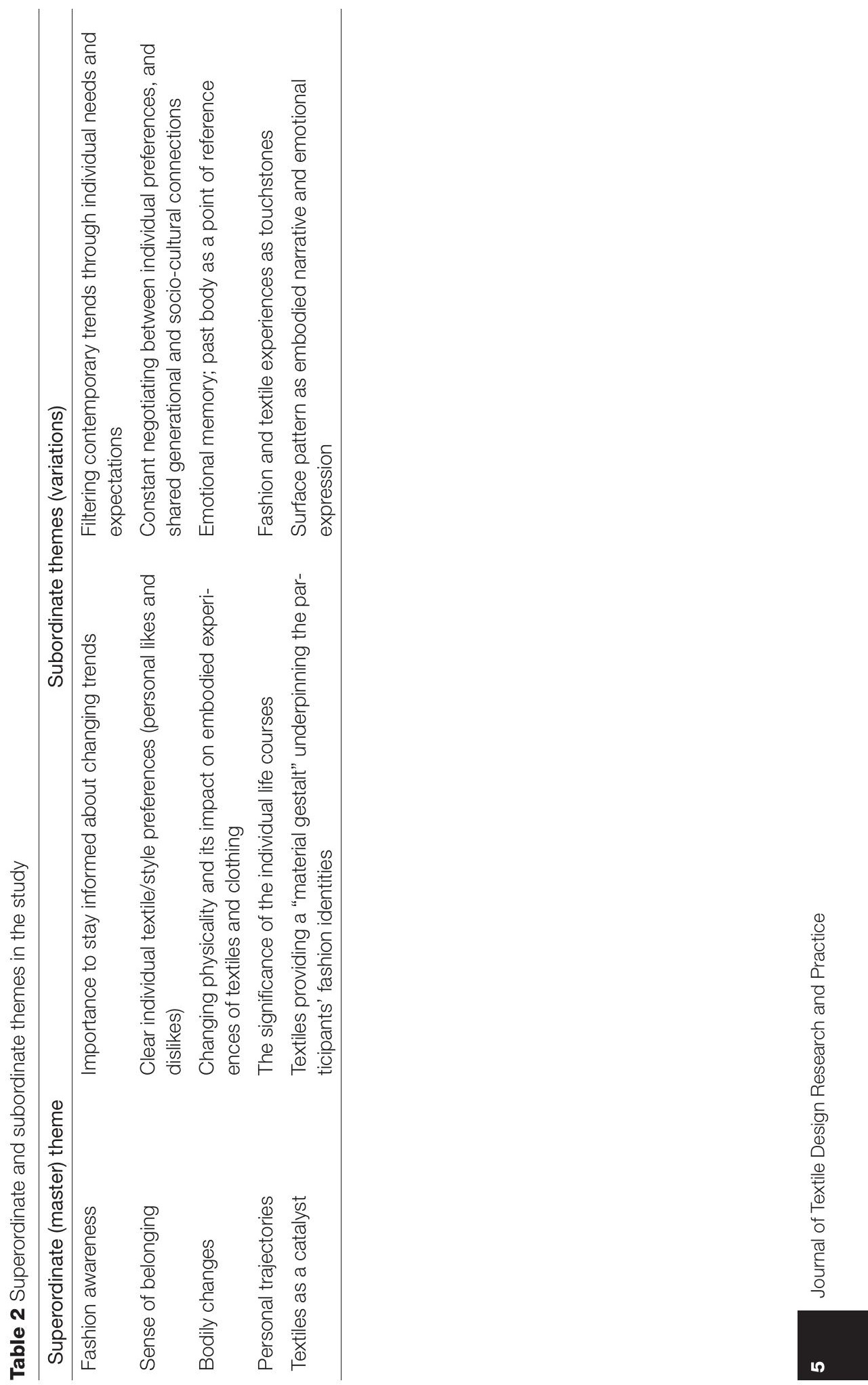


\section{Methodology}

The methodology employed within the Emotional Fit project (Townsend et al. 2017b) is informed by three complementary approaches: Interpretative Phenomenological Analysis (IPA), (Smith et al, 2009) participatory, co-design (Sanders and Stappers, 2008; Manzini 2015) and a simultaneous design method (SDM) for integrating the textile, garment and body form (Townsend 2004). Finlay (2011, p.140) identifies three "touchstones" of IPA: a reflective focus on individuals' subjective accounts of their experiences; an idiographic sensibility based on a close attention to the significance of particular lived experiences; and, the commitment to a hermeneutic approach which allows for a more interpretative understanding of the complexity and intricacy of human lifeworlds. In the case of Emotional Fit, this was closely aligned to the catalytic role that textiles have played in the process of fashioning the participants' identities. IPA methods included undertaking and analysing interviews and knowledge sharing workshops, allowing the researchers to accrue qualitative data pertaining to the life-course perspectives of the participants and to develop an in-depth understanding of their current experiences of fashion and clothing in relation to their past (Sadkowska et al 2016). Co-designing textiles and garments provided a "scaffold for supporting collective enquiry" (Sanders and Strappers 2008) drawing on "expert and diffuse" knowledge (Manzini 2015: 37) between all the stakeholders acting as collaborators in the crafting process (Ravetz et al 2013). The SDM method (Townsend and Goulding 2011: 308) builds on Sonia Delaunay's printed fashion practice (Damase 1991) through the simultaneous conceptualisation of $2 \mathrm{D}$ textiles, 3D silhouettes and the physiological profiles of the participating group.

Adopting a hybrid, practice-based methodology (Townsend 2004) over three phases (Table 1) created challenges and opportunities. Firstly, while both IPA and co-design allowed the researchers to develop an interpretative understanding of the participants' experiential fashion and textile knowledge, these approaches required a careful negotiation between the objectivity and subjectivity of those involved. This was especially significant when finding a balance between the participants' and researchers' individual and group preferences and expertise. Secondly, the adopted research methods facilitated the generation of rich research data, which encouraged online sharing methods in addition to using photographs, field notes, audio-visual recordings, and interview transcriptions.

\subsection{Tracing Participants Embodied Knowledge of Fashion and Textiles}

In the first phase of the study, a series of five in-depth, conversational interviews were conducted between June and July 2015 (Townsend et al. 2017b: 243) to establish participants' connections between the materials of dress and their individual agency (Malfouris 2013). A set 
of open-ended topics included: how the participants understood the concept of "fashion" and how they defined it; their past and present relationship with fashion, how it has changed over time; their perfect fashion item; their least favourite item; and, their clothing/dressing/wearing practices and habits. Probes and prompts were used to encourage elaboration on their "biographical wardrobes" (Skjold 2016).

The interviews were analysed using techniques standard to IPA with it's commitment to the detailed examination of how people make sense of their life experiences (Langdridge 2007; Smith et al. 2009; Finlay 2011; Sadkowska et al. 2016). These steps included: reading and re-reading of the interview transcripts; note taking; developing emergent themes; and searching for connections and patterns across cases. The final stage of analysis was devoted to clustering themes with contextual similarity, enabling the researchers to focus on developing a deeper level of interpretation. The outcomes included a series of five superordinate themes, with two subordinate themes each, describing the participants' experiences of fashion as they age (Table 2). In this article, we focus on 'Textiles as a Catalyst', comprising two sub themes: how textiles have functioned as material gestalt by underpinning their fashion identities; and the facility for surface pattern to trigger embodied narratives and emotional expression. All identifying information has been anonymized to protect the participants' privacy.

\subsection{Textiles as Material Gestalt for Underpinning Fashion Identities}

The narratives of the five interviewed women provided multiple insights into their embodied experience of fashion and clothing with textiles emerging strongly as a catalyst for various material gestalt, linked to narratives reinforcing product attachment and self-esteem (Niinimaki and Koskinen 2011). Interestingly, when asked about their personal understanding of the term "fashion", all of the participants described their past relations with clothing, often returning to their childhood and early teenage years, and explaining the importance of textiles as an enabler for acquiring certain homemade clothing skills (Twigger 2017b), inspiring their early interest in a fashionable attire. This was often linked to being part of the particular demographic of British women born in 1940 s and 1950s, who learned dressmaking from the previous generation (Goode 2016). In the following extract Barbara (65) explains how, being mentored by her mother offered her a space to learn dressmaking and develop a particular visual and tactile sensitivity to changes in fashion:

I was making my own clothes tutored by my mum just before that time [1960s] so I suppose I was also aware of what paper patterns were available, and initially I think they'd be quite classic in terms of what, fashion, you know, classic shapes and classic designs and then the kind of 60s hit and it was much more exciting and contemporary. 
Barbara's extract is notable for the juxtaposition of two descriptors: "classic" vs. "exciting and contemporary", the first being associated with the period prior to the 1960s socio-cultural revolution, equating to old-fashioned clothing; and the second, representing progressive styles. For Barbra, and most of the interviewees, there was a distinctive quality associated with the 1960s which symbolized newly gained freedom of creative expression, (Goode 2016; Sadkowska 2016). Barbara's reflection highlights an interesting overlap between two of the emergent superordinate themes, "Textiles as a Catalyst" and "Sense of Belonging" (Table 2) in the way that she carries fond memories of her youth and the clothes she made, purchased and wore. Another participant, Christine (65), offers similar reflection on the importance of learning dressmaking skills from her mother correlated with her unique body form, and ability to create garments that were customised to her needs:

I've always had an interest in clothing which is I think because I've always been a little bit plump and quite short so not always fitting into clothes, even from a young age, and because I liked sewing and my mother was able to teach me and encourage me l've always made clothes and I've always liked fabrics and textiles.

For Christine, using textiles as a way to clothe her individual body shape in an informed and responsive way, was linked to her developing a sense of achievement in fashioning herself, as she explained elsewhere in the interview: "Because over the years, I feel that my body type is a bit difficult and so therefore l've probably worked out how to dress it". Dant (2007: 383) notes that it is "the engagement of the wearer with the garment such that they become part of each other" that gives the materiality of clothes it's meaning. With Christine this engagement was threefold: firstly by making clothes with her mother; secondly by wearing them; and thirdly by working out the best way to clothe and present herself (Entwistle 2002). In this way the cut, drape and the selection of the fabric itself all reveal aspects of its unfolding meaning to Christine. Another participant, Elizabeth (67) frequently comments on her short stature and related clothing limitations, also reflecting on her interest in fashion as being closely linked to finding clothes that suit her particular figure: "So all the way through I suppose I have been kind of interested in clothing rather than fashion".

These extracts illustrate that from a very early age, textiles facilitated a creative space for the participants, where by producing their own clothing they developed an intimate understanding of their unique body forms. Twigg (2013: 12) notes that the way clothes are constructed "reflect assumptions about the sort of bodies that will inhabit them, so much that these can be read across from the physical objects to those who will or did occupy them". In terms of the study participants this can be interpreted as a specific type of (self-)tacit knowledge of "discover- 
ing their identity or identities through a process of understanding and interpreting their own responses to the various styles brought to their attention" (Kent 2017: 19). Additionally, the participants often linked the materiality of textiles with particular memories based on sensorial feelings and self-esteem based on making their own clothes.

\subsection{Surface Pattern as Embodied Narrative and Emotional Expression}

The second subordinate theme is based on the propensity for textile patterns to trigger personal narratives and emotional responses. This theme was present in nearly all the interviews, emerging as complex and multi-layered in terms of how the story telling about current and past garments were dominated by descriptions of the textiles, with strong references to their colours, patterns, embellishment, and tactility.

In the following extract, Anna (64) explains how textiles became a catalyst for her producing particular outfits:

I make my own clothes for special occasions, for weddings or special occasions I certainly do. And then if I just find some nice fabric, I go on EBay and buy some nice saris in silk and make them into jackets and tops.

The repeated use of the adjective "nice" here in this otherwise short extract, is notable, whereby the carefully selected fabric allows Anna to create an outfit she is happy with in a particular context. A similar reflection is presented by, Barbara (65):

I do think of natural fabrics as beautiful, yes, wool, cotton, linen, yes, I went on holiday when I was four months pregnant with my first child 33 years ago in Italy, and went into a fabric shop (...) and I bought some black linen there that was, oh, just fantastic, (...) when I say shiny it wasn't a high shine, but there was something, sheen was just part of the quality - it was just fantastic quality linen, and I, I made myself a suit from that, culottes, and a boxer jacket, (...) I felt really good wearing that linen, (...) I do remember the attention that wearing that suit attracted which was great.

Barbara's account revolves around descriptors of "beautiful", "fantastic", "quality", "good", and "great", which convey how positive the experience of making and wearing those garments was for her. The extracts above, suggest that for these interviewees it was the process of selecting the cloth and making the garments that catalysed a particular emotional durability, and a long lasting relationship with them (Twigger Holroyd 2017a). Similarly, Chapman (2014: 137) argues that 
"[a]lthough we may assign particular meaning to a given object, or material, meaning cannot exist outside the body. [And it] cannot hold meaning in and of itself - only our interpretation of these things will produce meaning".

As explained further in the article the idea of facilitating and sustaining a strong psychological connection between the maker and the wearer (enacting interpreter) of the textiles as well as the garment, by blurring the two roles, became an important element within the collaborative designing process Townsend et al. 2016.

Another layer of this subordinate theme is present in the following extract from Anna, where she cites Zhandra Rhodes to contextualise her understanding of the fashion system as a cycle of constant changes, and to locate herself within it.

She [Zandra Rhodes] has always been cutting edge and still is I think, right from the start. But with colour, you know it's the colour that drives... all the different textures, embroidery and beads, we keep reusing the same ideas don't we? (...) [And] I think when you get older it's easy to just become a sheep in a flock or sheep with grey hair and tweed skirts, I don't want to be like that, I want to be individual. And I think it is more important once you get over fifty and you know your colour starts going and people tend to sort of, you become invisible because you are old and that is why I like Zandra Rhodes.

Rhodes represents the baby boomer generation and as a designer is known for her intricate and often dramatic textiles. So Anna's fashion choices are meaningful in terms of the colours and patterns she wants to be associated with, refusing to blend in or become invisible. Instead, by selecting the right textiles she can potentially re-live the excitement and attention she experienced from wearing Rhodes designs in the past. As Featherstone and Hepworth observe in The Mask of Ageing (1991) as the body ages, and the physical changes occur, there is a growing discrepancy between the enactment of what individuals might aspire to and what is presented. Twigg (2013: 14) implies that "the discordance is not total, and the promised renewal continues to work, but to a lesser degree and with disquieting subtexts", which explains some of the frustrations that our participants shared with us.

Finally, this subordinate theme is concerned with textiles functioning as a catalyst for a visual and emotional transformation. While the potential of fashion and textile design is especially potent in terms of enhancing and enriching our lives (Gwilt 2015), this theme emerged as complex, multifaceted and open to various interpretations. Elizabeth offers a particularly moving account of how emotional durability is the result of the close psychological connection she makes between a special garment and her husband: 
The (...) thing I felt really good in was, it was in Vietnam and it was in this kind of little village and there was this tribe. They were selling kind of little hand-embroidered jackets and of course they are all small in Vietnam, the women. It was dark navy and it had this beautiful kind of blues and greens embroidery. [My husband] said oh that's nice. I just went and bought it on sight. And in fact I was told their colours run so I kind of soaked it in salt. Oh I loved that jacket. I would wear it for Christmas for do's and I actually wore it for the funeral [of my husband] because that was always something that I was really happy to wear. (...) I loved that jacket.

What is significant in Elizabeth's account is the way in which the materiality of the garment, its colour and artisanal embellishment, together with the history of purchasing it while accompanied by her late husband allows her to find comfort in wearing it. While such emotional attachment is closely linked to the participants' personal trajectory (Lerpiniere 2015) and, consequently is difficult to artificially replicate within the design process, it was through the method of wardrobe inventories that some of the elements, such as specific motifs, colour ways or patterns, were captured as meaningful textile narratives (Townsend 2011) and influenced the aesthetics of the co-designed artefacts.

\section{The Interrelating of Print, Fabric and Form}

As suggested in the interviews, growing up in the 1960s and 1970s, most of our participants were proactively involved in making, customizing and repairing their own clothes (Goode 2016; Twigger Holroyd 2017a, 2017b). Fashion designers such as Biba, Laura Ashley, Mary Quant, Ossie Clark and Zandra Rhodes were referred to throughout the project, in relation to their influence on the style of contemporary silhouettes and corresponding textile designs and qualities. Notably, all these practitioners designed their prints themselves, or in-house and their practice represents three different approaches to how the role of textiles can act as a catalyst in the fashion design process though examples of "architectural", "sculptural" and "crossover" (or hybrid) models (Townsend and Goulding 2011: 295-300). For example, Quant's short, geometric shapes were often realised in bold, graphic printed, woven or knitted qualities that echoed the boxy silhouettes. While the wearer's arms and legs were often revealed, the body (shoulders and waist) acted as an armature for the architectural dress forms (Townsend and Goulding 2011: 299). In contrast, Clark integrated Celia Birtwell's feminine, floral prints on the straight-of-grain and bias, aligning the imagery with the silhouette and underlying contours via body sculpting forms. Rhodes oversized kimono shapes in vibrant printed silks and voiles represent a hybrid of the architectural and sculptural archetypes freeing the body completely in a diaphanous shape or drawing attention to certain areas, using decorative ties and details. The designer's method of building up the geometric garment shapes with printing screens 
represented a complex "textile-led" approach to fashion that preempted integrated CAD technology (Townsend and Goulding, 2011: 304).

Having considered these printed garment design archetypes, the team selected the "hybrid" model represented by the work of Zandra Rhodes, by aiming to develop styles that facilitated "elegance, comfort and openness" and where "the body shapes the clothes more than the clothes shape the body" (Van Essche quoted in Aakko 2016: 187). At the start of the creative workshops a "simultaneous design method" (SDM) (Townsend 2004) representing a digital interpretation of Delaunay (and Rhodes') conception of the "the cut and decoration of the dress" being developed concurrently (Damase 1991: 58) was adopted. However, due to the number of co-designers (researchers=n5; participants $=\mathrm{n} 45$ ) and the research and development being carried out over a two year period, this approach was necessarily augmented by a "parallel" version (Piper and Townsend 2015) whereby the prints were designed alongside and in response to the emergent garment silhouettes and feedback from the participants.

\subsection{How Textile Preferences Informed Garment Silhou- ettes}

The empirical approach to gathering insights into the lived fashion experiences of our participants resulted in rich textile and clothing data for semiotic analysis (Woodward and Fisher 2014: 5). Key words identified within the "textiles as catalyst" and related themes included references to "natural fibres" and material-based adjectives such as "comfort", "high quality", "flexibility", "ethical sourcing" and "sustainability". Based on these clues different weights of woven and jersey fabrics were selected to inform initial shape making with rectangular and square-based toiles utilizing the full width of the cloth, as shown in Figure 2. Led by Sissons, the researchers measured and conducted live draping on the participants, primarily to inform scale and silhouette, but leading to further insights into individuals embodied knowledge of fabric, how they preferred to "reveal and conceal" and "invent and create" themselves through different "folded and unfolded" creations (Barnett 1999; 25). Experimenting with "the space of surface and texture, material and matter: the physical stuff from which things are made" (Barnett 1999: 29) was instrumental throughout the co-designing process in recognition that:

New forms can emerge when exploring body movements and identifying properties of comfort that occur in bodily interactions between people and their environments, especially when observing how a body is shaped and patterned, or how people wrap their bodies as part of their wearing behaviours. (Jeon 2015: 149) 

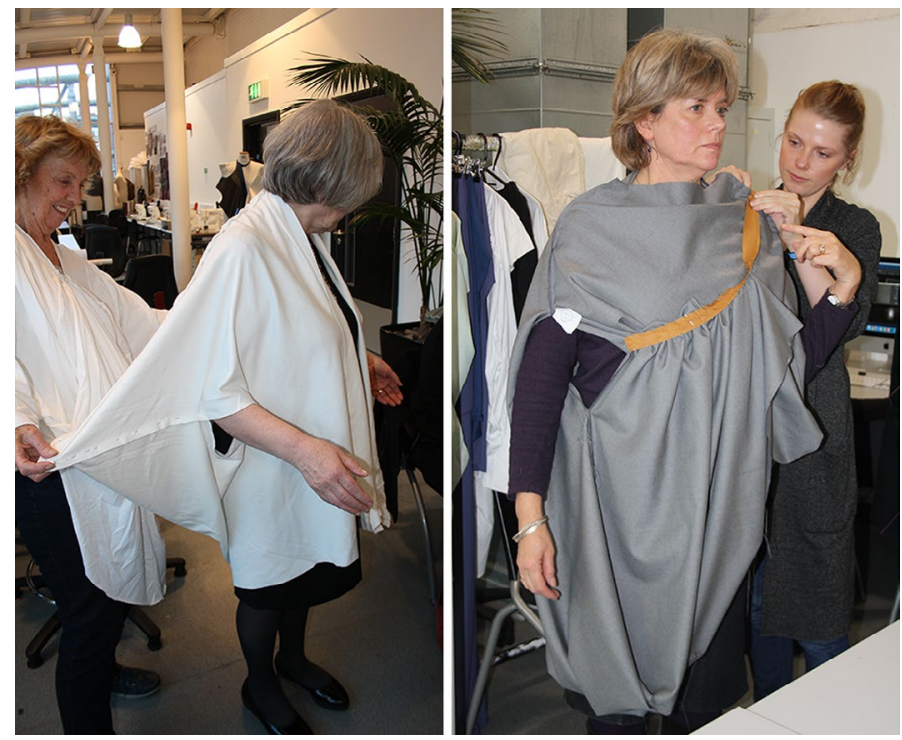

Figure 2

Initial experiments using live draping using jersey and woven qualities and the whole width of the cloth. (c) Emotional Fit 2015.

Geometric shapes were cut on the straight-of-grain and bias to investigate the fit and drape of these potential garment silhouettes. A "jigsaw" (Rissanen and McQuillan 2015: 85) approach accommodated tessellated triangles and circular shapes where spare fabric was allocated for pockets and bindings. Heavier weights were utilised for garments with less volume, providing a quality drape that does not cling to every curve of the body as explored via the circular toiles (Figure 3). The lighter jersey drapes and woven wool and silk were used to develop fuller shapes, including the Triangle and Rectangle jersey dress illustrated in Figure 4, providing greater scope for fabric manipulation and sculptural styling (Sissons 2010: 54). Overall, the preferred finishing style was minimal, including the use of raw cut edges, revealed seams and garment ties, more associated with the material qualities of a "deconstructed approach" that conveyed how the garment had been made (Townsend 2011).

Adopting geometric pattern cutting offered possibilities for creating minimal, versatile silhouettes with a similar aesthetic to traditional garments such as the sari, kaftan and kimono, reinforcing references made by the interviewees. These universally worn and recognized shapes also facilitated multiple patterning and flexible styling options in line with the "artisanal" or "in-between" fashion (Aakko 2016) produced by independent, craft based designers. 

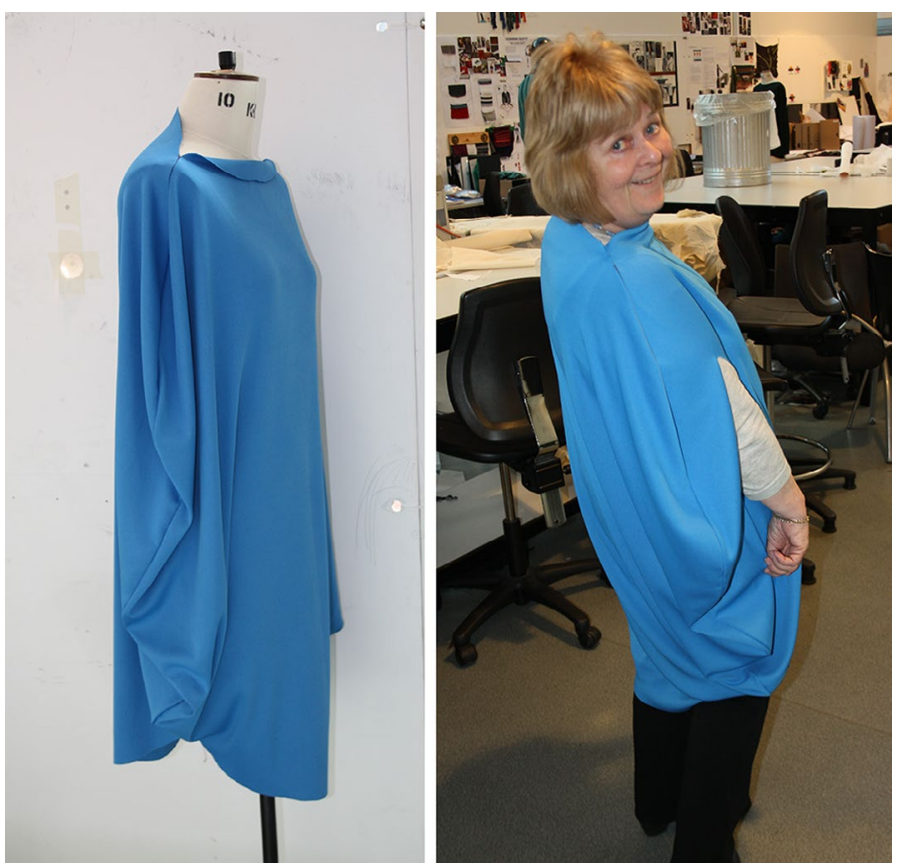

Figure 3

Geometric shape development with heavy jersey to produce a circular toile on the stand and body form. (C) Emotional Fit 2015.

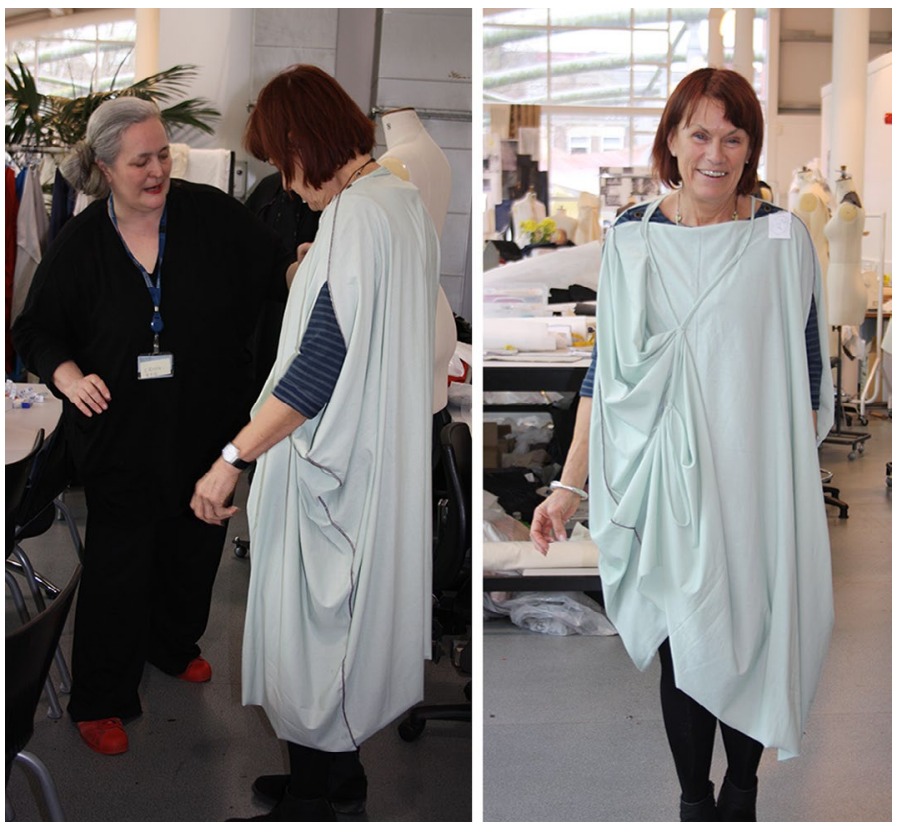

Figure 4

Modeling lightweight jersey on one of the research participants to produce a manipulated, rectangular toile. (c) Emotional Fit 2015. 


\subsection{Connecting Past and Future Textile Narratives through Print}

The relationship with the body is generally more direct in fashion, than in textiles (Townsend and Goulding 2011: 289). However, in this research print designs were configured in parallel with live draping, garment shape development and the narratives of the participants. In order to develop solutions that responded to the women's past and evolving design aspirations, visual and tactile qualities were considered in terms of the group's shared and individual fashion histories, and how they wanted garments to interact with their bodies and current lifestyles. This disentangling of human and material agency was extremely challenging, involving symbiotic analysis of the intentional and casual interactions involved in fashion and dressing (Malafouris 2013; Entwistle 2015 \& 2002). Consequently, experimental prints were developed by identifying emotional signifiers of textile attachment through making, owning and maintaining particular items of clothing. A series of fabric swatches were designed and printed based on original photographs of natural phenomena found in domestic, urban and rural environments, as identified by the participants as "everyday contexts". Striped, floral and abstract patterns, popular in the 1960 s and 70s were in favoured tertiary colours, including dusky purple, indigo, rust, khaki and dark grey. These initial ideas (Figure 5) were inkjet-printed using reactive dyes on organic cotton, viscose, silk, wool, linen and jersey qualities, with each design transposed on three contrasting substrates to produce over 30 samples for the participants to select from.

The printed textiles were reviewed and ranked by the participants in order of preference, using feedback sheets requiring a mark between 1-10 and comments. The results were analysed and the most popular designs developed as lengths to be combined with some of the most tried-on garment shapes, such as the Triangle and Rectangle Dresses. Fundamentally, it was the tactile qualities and colour ways that influenced the womens choices more than the imagery, for example, one participant stated she "would never wear anything like that" (on linen) then selected the same design printed on silk as her "favourite". This intuitive prioritisation of colour and texture over graphic composition was reiterated by other participants, highlighting the perpetual phenomenon of synaesthesia; how the "the eye, one sense organ among others, does not simply look. It also feels" (Barnett 1999: 28).

Some of the most significant interactions between the group members occurred in Workshops 3 and 4 where participants used their intuitive textile knowhow based on preconscious material experiences (Chapman 2014: 138) to select and combine toiles and prints (Figure 6) through play and trying things on, as explored in the installation, Trying it On (Townsend et al. 2017a). Here, the speculative nature of the partially materialised objects allowed for unexpected interventions and insights into the value of the collaboration as a "creative journey" as opposed to "a means to an end" (Millar in Ravetz et al. 2013). For example, the toiles and swatches were handled spontaneously and knowingly, in a similar unspoken manner as when someone peruses garments on a rail using visual and tactile perception, or gestalt, before selecting one to 


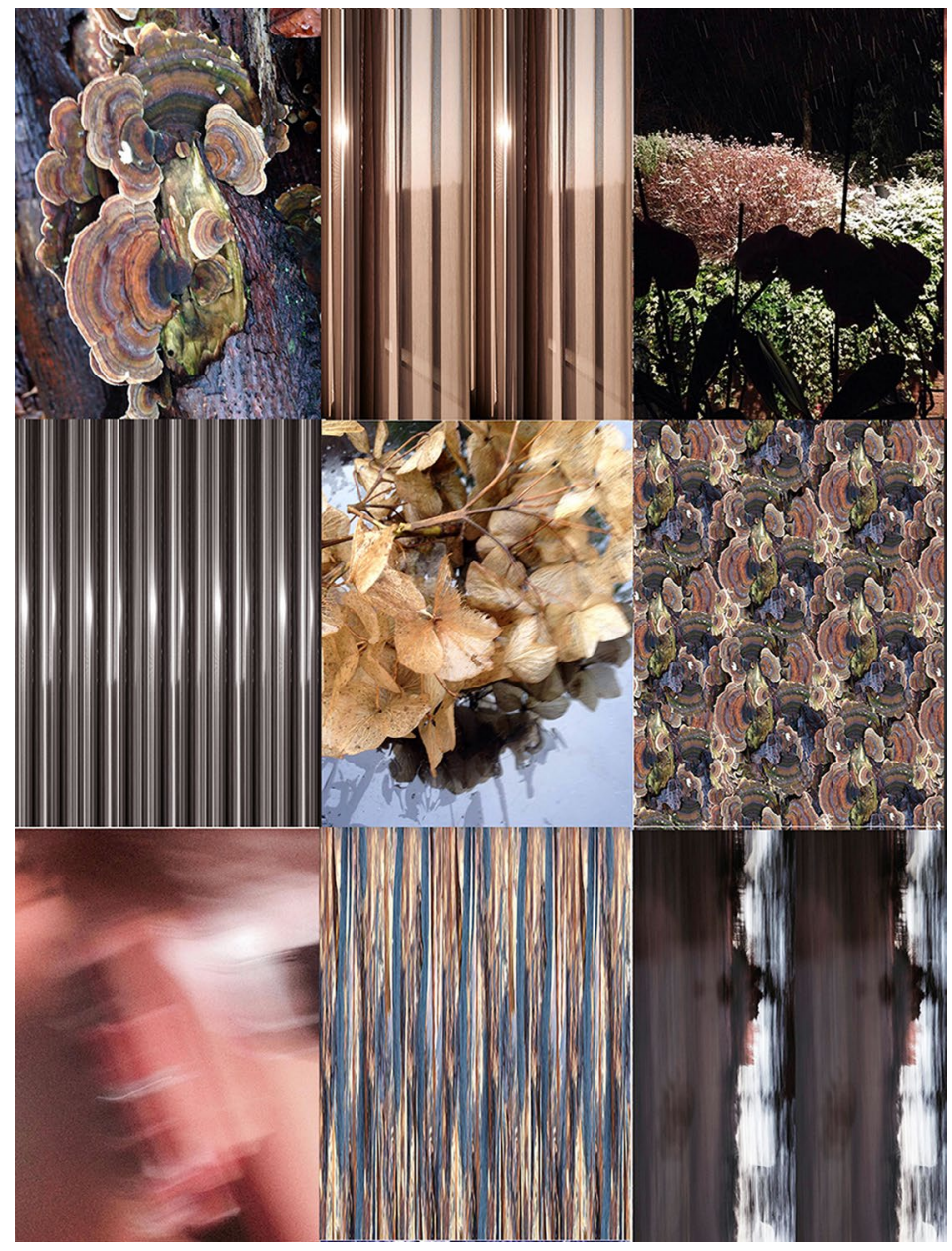

Figure 5

Nine initial print ideas featuring imagery, patterns and colours in response to interview findings and workshop discussions. (c) Emotional Fit 2016.

"try on" (Townsend et al. 2017a). The unfinished nature of the artefacts encouraged the participants to demonstrate their knowledge of fashioning, by enacting a kind of "hacking" as described by Busch:

[But] hacking can also be used to mean the reclaiming of authorship (or co-authorship) of a technology by supporting transparency and unanticipated use... hacking is a critical as well as playful activity, circling around a DIY ethos and a desire to amplify our means of interaction with the world. (von Bush 2009: 165)

The reintroduction of the body into the fashion process was reciprocated via personalization through folding, stretching and cutting to reduce or extend the silhouette and prints being compared, and animated to mimic and assess drape and performance. Here, the 


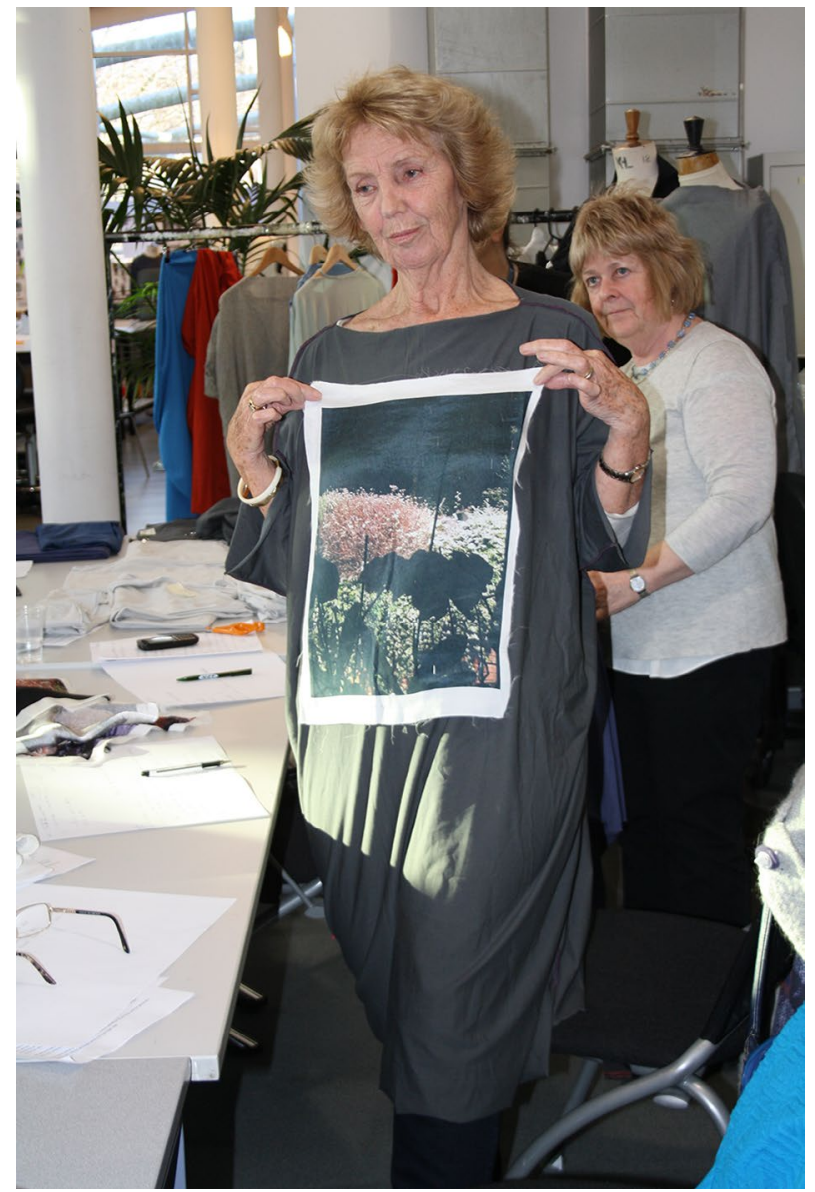

Figure 6

One of the research participants trying on a toile and selecting a complementary printed textile. (c) Emotional Fit 2016.

"body itself became an essential structure in crafting the form of the textile design" (Jeon 2015: 138). It was the participants "sensing" of the research materials using their "embodied fashion knowledge" that most significantly influenced the development of the prototypes. The approach unified meanings associated with the different kinds of materiality (and immateriality) inherent in "objects of fashion", clothing items kept as "treasured possessions" and "virtual" representations of fashion - "which are consumed as part of the process of identity formation through fashion" (Woodward and Fisher 2014: 9).

\subsection{Engineering and Constructing Printed Textiles as "research tools"}

Following the print selection process, the surface imagery was experimented with in CAD by engineering the scale, direction and placement in relation to the geometric $2 \mathrm{D}$ pattern pieces and by assessing the 
overall 3D drape of the silhouette. For example, a Stripe design based on a photograph of light permeating drapery was adjusted (in Adobe Photoshop) to correlate aesthetically with three different (grouped) sizes of garment; Small (8-12); Medium (12-16) and Ample (16-20). The overlapping sizes of the pattern pieces reflected the versatile nature of "fit" required by the women (Townsend et al. 2017b: 247). The Stripe print was manipulated to visually contour both the garment and underlying form of the wearer by repeating and stretching the vertical elements across the total $(145 \mathrm{~cm})$ width of the fabric, at three varying scales and tones; generating three contrasting iterations from narrower and lighter to wider and tonally darker. The digital crafting approach resulted in each garment having a unique visual effect on the body, achieved by the team considering the aesthetic and physical attributes of print, garment and body simultaneously (Townsend 2011: 288).

The Stripe design was also mediated through a strategic cutting approach. Although zero and minimal waste were prioritised, the geometric shaped pieces of the Triangle Dress, allowed for varying arrangements to be explored through the construction process, while adhering to the straight-of-grain. The dual aim of sustainable cloth consumption while creating a garment with a bespoke aesthetic, was facilitated via a combination of jigsaw lay planning, optical prints, patch-working and trompe l'oeil pleat effects (Townsend and Goulding 2011). Figures 7 and 8 show different print configurations on two dress fronts. This hand/digital method also draws on Piper's Composite Garment Weaving method where the consideration of 2D "functional" textile production aspects strongly inform the "design aesthetic" of the final 3D object (Piper and Townsend 2015: 18).

The Triangle Dress was also fabricated in Orchid printed silk, which was scaled according to the three grouped sizes and cut on the more

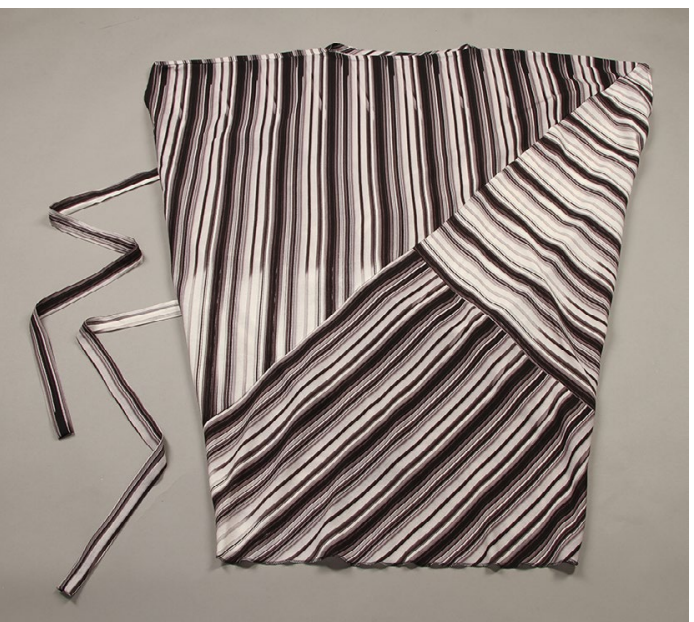

Figure 7

2D version of the Medium Striped Triangle Dress showing configuration of pattern within the garment shape. (c) Emotional Fit 2017. 


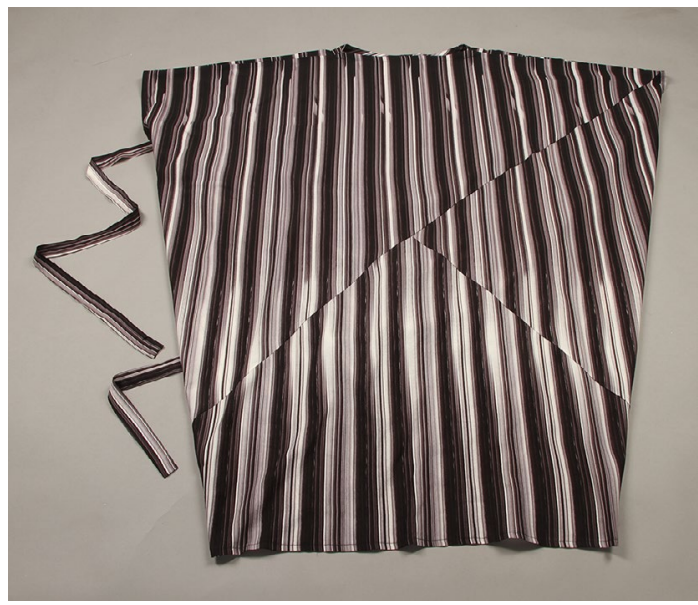

Figure 8

2D version of the Ample Striped Triangle Dress showing different configuration and tonal range of pattern than the Medium version (shown in Figure 7). (C) Emotional

Fit 2017.

subtle, reverse of the cloth following feedback from the participants. While both the Stripe and Orchid versions of the dress presented distinct visual and material aesthetics, each incorporated "empathic design" features, such as long ties, pockets, reversibility (both the textile and garment could be worn back to front) allowing for multiple styling options to enhance textile and "product attachment" (Niinimaki and Koskinen 2011). Figures 9 and 10 illustrate the divergent material aesthetics achieved through similar silhouettes.

\section{Discussion}

By co-creating printed garment prototypes based on feedback from potential consumers the project contributes to the expansion of the concept of "lead users as collaborators" in fashion design and personalisation (Morris and Ashdown 2018; Kent 2017). This article has focused on how textiles played an instrumental role within a collaborative project, which aims to raise awareness of older consumers needs and motivations in relation to fashionable dress. Interviewing and talking to the women in workshops about their personal fashion histories, then analysing these accounts, using IPA methods, resulted in rich qualitative data relating to the role and value of textiles within their lives. The narratives informed the project on an aesthetic, technical, sustainable but ultimately a human level; the final outcomes shaped by "alternative understandings of immaterial culture" (Chapman 2014: 137) as well as direct engagement with materials. The process of creative exchange reminds us of how "The fashion system not only provides garments for wear, it endows garments with beauty and desirability ... In doing so, it weaves aesthetics into the daily practice of dressing" (Entwistle 2015: 43). 

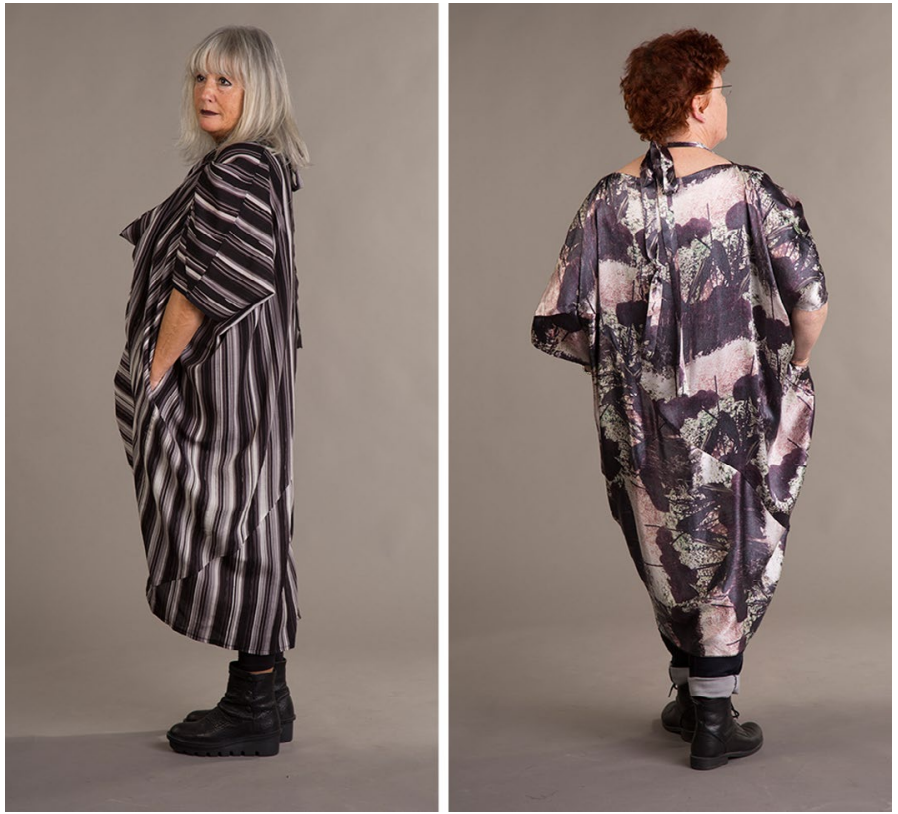

Figure 9

Striped and Orchid Triangle Dresses modelled and styled individually by two of the research participants. (c) Emotional Fit 2017.

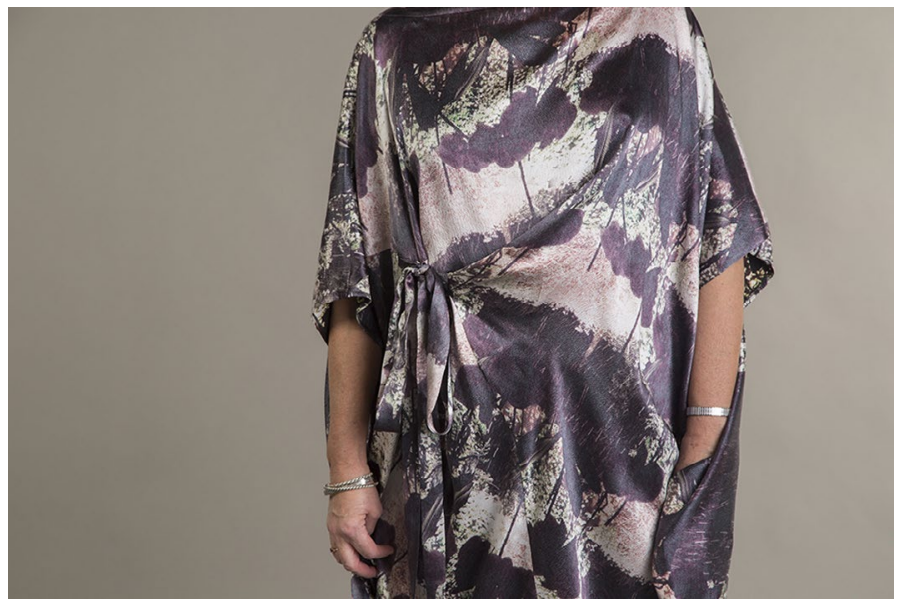

Figure 10

Close up of the kimono style Orchid print Triangle Dress showing it tied at the front and cut on the reverse of the original fabric in response to feedback from some of the participants. (C) Rebecca Lewis 2017.

Materiality in the context of aesthetics also implies connection to the body. Above all clothing serves function, which generally drives many aesthetic choices. Functionality aside, in this case 
materiality refers to the design process and the relationship of the garment with the body. (Aakko 2016: 187)

The simplicity and versatility of the triangular, rectangular and circular cut pieces invited experimentation and agency through play, identified as often missing from the high street. The concept of personalising universal shapes reinforces the need for methodologies that integrate more sustainable product relationships through closer connection between society and the practices of design and making (Niinimaki and Koskinen 2011; Twigger Holroyd 2017b). The prototypes developed through the project represent "research tools" that can lead to personalisable dress objects that more closely incorporate the values of a new kind of "fashion-in between", represented by independent, artisanal and high quality design, materials and craftsmanship (Aakko 2016). The employed methodology focuses on the significant role that textiles can play, as both catalyst and scaffold, within the process of collective creative enquiry (Townsend et al. 2016). While technology continues to shift the design process closer to future users (Sanders and Strappers 2008), there are surprisingly limited examples of empathic design initiatives that seek to harness deeper understanding of consumers emotional needs (Niinimaki and Koskinen 2011). Socio-material engagement holds considerable untapped potential for value creation, through the adaptation of a more open source, less hierarchical fashion system that draws on the creative craftsmanship engendered by software related "hacking and networking" models (von Bush 2009). Figure 11 shows some of the group members during a trying on session and photo shoot by Rebecca Lewis.

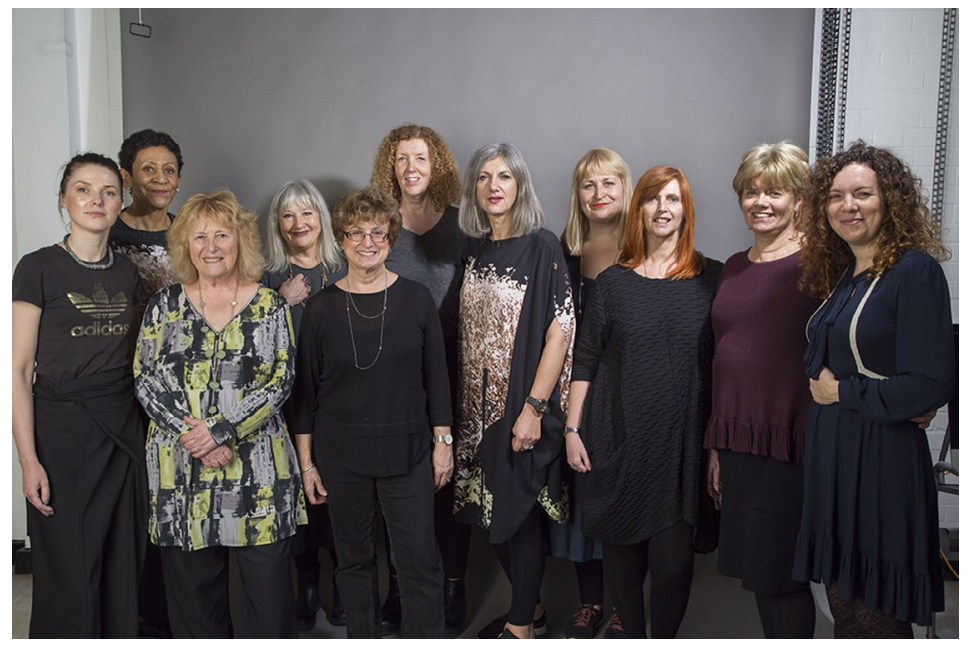

Figure 11

Some of the members of the Emotional Fit research team. (c) Rebecca Lewis 2017. 


\section{Acknowledgements}

We would like to thank our research collaborators, Juliana Sissons, Katherine Harrigan, Katherine West and all the women from Nottingham involved in the project.

\section{Funding}

This reasearch was supported by the School of Art and Design, Nottingham Trent University.

\section{ORCID}

Katherine Townsend (D) http://orcid.org/0000-0003-2212-2511

\section{References}

Aakko, M. 2016. Fashion In-between: Artisanal Design and Production of Fashion. PhD thesis. Helsinki: Aalto University.

Barnett, P. 1999. "Folds, Fabrics and Surfaces: Towards a Poetics of Cloth." In Binns, P., Barnett, P. and Johnson, P. (Eds), Exhibition Catalogue for Textures of Memory: The poetics of cloth, pp. 24-34. Nottingham: Angel Row Gallery.

von Bush, O. 2009. "Engaged Design and the Practice of Fashion Hacking: The Examples of Giana Gonzalez and Dale Sko," Fashion Practice, 1(2): 163-185.

Chapman, J. 2014. "Designing Meaningful and Lasting User Experiences." In A. Moran and S. O'Brien (ed.) Love Objects: Emotion, Design and Material Culture, pp. 137-148. London: Bloomsbury.

Damase, J. 1991. Sonia Delaunay: Fashions and Fabrics. London: Thames and Hudson.

Dant, T. 2007. "Consuming or Living with Things?/Wearing It Out." In M. Barnard (ed.) Fashion Theory: A Reader, pp. 373-385. London: Routledge.

Emotional Fit. 2017. Documentary Video (10 mins). Nottingham: Jim Boxall, School of Art and Design, Nottingham Trent University.

Entwistle, J. 2002. "The Dressed Body." In M. Evans and E. Lee (ed.) Real Bodies: A Sociological Introduction, pp. 133-150. Basingstoke: Palgrave.

Entwistle, J. 2015. The Fashioned Body: Fashion, Dress and Modern Social Theory, 2nd ed. Cambridge: Polity Press.

Featherstone, M. and Hepworth, M. 1991. "The Mask of Ageing and the Postmodern Lifecourse." In M. Featherstone, M. Hepworth and B. Turner (ed.) The Body: Social Process and Cultural Theory, pp. 170-196. London: Sage.

Finlay, L. 2011. Phenomenology for Therapists: Researching the Lived World. Oxford: Wiley-Blackwell.

Gilleard, C. and Higgs, P. 2014. Ageing, Corporeality and Embodiment. London: Anthem Press. 
Goode, J. 2016. "Fashioning the Sixties: Fashioning Narratives of Older Women," Ageing and Society, 1-21, August 2016, Cambridge University Press.

Gwilt, A. (ed.) 2015. Fashion Design for Living. London: Routledge.

Jeon, E. 2015. "Form Empowered by Touch, Movement and Emotion." In A. Gwilt (ed.) Fashion Design for Living, pp. 134-150. London: Routledge.

Kent, T. 2017. "Personalisation and Fashion Design." In I. Kuksa and T. Fisher (ed.) Design for Personalisation. London: Routledge.

Langdridge, D. 2007. Phenomenological Psychology: Theory, Research and Method. London: Pearson.

Lerpiniere, C. 2015. The Textile Archive: Curating Personal Histories and Family Narratives. PhD thesis. Leicester: De Montfort University.

Malafouris, L. 2013. How Things Shape the Mind: A Theory of Material Engagement. Cambridge, Massachusetts and London, England: The MIT Press.

Manzini, E. 2015. Design, When Everybody Designs: An Introduction to Design for Social Innovation. Cambridge, MA: The MIT Press.

Morris, K. and Ashdown, S. 2018. "Expanding the Concept of Lead Users as Collaborators in Functional Apparel Design," Clothing and Textiles Research Journal.

Niinimaki, K. and Koskiken, I. 2011. "I Love this Dress, it Makes me Feel Beautiful: Emotional Knowledge in Sustainable Design," The Design Journal, 14(2): 165-186.

Piper, A. and Townsend, K. 2015. "Crafting the Composite Garment: The Role of Hand Weaving in Digital Creation," Journal of Textile Design Research and Practice, 3(1-2): 3-26.

Ravetz, A., Kettle, A. and Felcey, H. 2013. Collaboration through Craft. London: Bloomsbury Academic.

Rissanen, T. and McQuillan, H. 2015. Zero-Waste Fashion Design. London: Fairchild Books/Bloomsbury.

Sadkowska, A. 2016. Arts-Informed Interpretative Phenomenological Analysis: Understanding Older Men's Experiences of Ageing through the Lens of Fashion and Clothing. PhD thesis. Nottingham: Nottingham Trent University.

Sadkowska, A., Wilde, D. and Fisher, T. 2016. "Third Age Men's Experience of Fashion and Clothing: An Interpretative Phenomenological Analysis," Age, Culture, Humanities: An Interdisciplinary Journal, 2(1): 34-68.

Sanders, E.B.-N. and Stappers, P.J. 2008. "Co-creation and the new landscapes of design," CoDesign, 4(1): 5-18.

Sissons, J. 2010. Basics in Fashion Design 06: Knitwear. London: AVA Publishing.

Skjold, E. 2016. "Biographical Wardrobes: A Temporal View on Dress Practice," Fashion Practice, 8(1): 135-148.

Smith, J.A., Flowers, P. and Larkin, M. 2009. Interpretative Phenomenological Analysis: Theory, Method and Research. London: Sage.

Townsend, K. 2004. "Transforming Shape: Hybrid Practice as Group Activity," The Design Journal, 7(2): 18-31. 
Townsend, K. 2011. "The Denim Garment as Canvas: Exploring the Notion of Wear as a Fashion and Textile Narrative," Textile: The Journal of Cloth and Culture, 9(1): 90-107.

Townsend, K. and Goulding, R. 2011. "The Interaction of Two and Three Dimensional Design in Textiles and Fashion." In A. BriggsGoode and K. Townsend (ed.) Textile Design: Principles, advances and applications, pp. 288-322. Cambridge: Woodhead Publishing and The Textile Institute.

Townsend, K., Sadkowska, A., Goode, J., Sissons, J., Harrigan, K. 2016. "Design as a Socio-Material Practice: Reflections on the Emotional Fit Collective Fashion Enquiry." Everything and Everybody as Material, 7 - 9 June, The Swedish School of Textiles, University of Boras, Sweden, pp. 190-199, Available at: http://media.everythingeverybodyasmaterial.com/2017/12/EEM-PROCEEDINGS-2017. pdf

Townsend, K., Sadkowska, A., Sissons, J., Harrigan, K., West, K. and Boxall, J., 2017a. Trying it On. Intersections exhibition: collaborations in textile design research, Loughborough University London, London, 13-14 September 2017, p.28, Available at: https://www. Iboro.ac.uk/media/wwwlboroacuk/external/content/schoolsanddepartments/aed/downloads/INTERSECTIONS2017_EXHIBITION_ CATALOGUE.pdf

Townsend, K., Sissons, J., Sadkowska, A. 2017b. "Emotional Fit: Developing a New Fashion Methodology with Older Women," Clothing Cultures, 4:3, pp. 235-251. Bristol: Intellect.

Twigg, J. 2013. Fashion and Age: Dress, the Body and Later Life. London: Bloomsbury.

Twigger Holroyd, A. 2017a. "From Stitch to Society: A Multi-Level and Participatory Approach to Design Research," Design Issues, 33(3): 11-24.

Twigger Holroyd, A. 2017b. Folk Fashion: Understanding Homemade Clothes. London: I. B. Tauris.

Woodward, S. and Fisher, T. 2014. "Fashioning through Materials: Material culture, Materiality and Processes of Materialiization," Critical Studies in Fashion and Beauty, 5: 1, Intellect. 